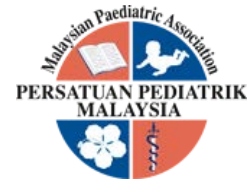

\title{
NEURO-ENTERIC DYSFUNCTION IN CHILDREN WITH SEVERE NEUROLOGICAL IMPAIRMENT
}

\author{
Fahisham Taib ${ }^{1,2}$, Nik Zainal Abidin Nik Ismail ${ }^{1,2}$
}

\begin{abstract}
Visceral hyperalgesia, intestinal dysfunction and unexplained irritability in neurological impaired children is a medical enigma for many healthcare professionals. The neuro-medical management can be challenging and difficult, due to poor understanding of the underlying aetiology and pathophysiology of the condition. Neuro-enteric axis has been proposed as emerging physiologic mechanism in the pathogenesis of many gastrointestinal diseases. The bidirectional connection between enteric and central nervous system may represent a direct relationship between neurological system and gut physiology. Insult to the brain indirectly contribute to the ongoing gut and brain axis sequalae. Microbiota has been an important modulator in the brain-gut axis. Irritability episodes in severe neurological impairment children has been commonly associated with pain originated from gastrointestinal pathology. Management of such condition requires a holistic approach to tackle multidimensional factors that has contributed to the 'totality' of the symptoms.
\end{abstract}

\section{Keywords:}

Brain-Gut Axis, Neurology, Children, Neurological Impairment, Gut Dysfunction

\section{Introduction}

Parent commonly associated pain in neurologically impaired children with the gastrointestinal disorders. For various reasons, these children are often irritable and difficult to manage. Neuro-enteric dysfunction is seen in severe neuro-disability conditions as a result from combination between oromotor dysfunction, communication difficulties and motor impairment, leading to faltering in growth [1]. The relationship between gastrointestinal microbial pathogen and brain arose from observation of patients with hepatic encephalopathy who improved following administration of oral antibiotics [2]. Microbiota was thought to have a role in conditions such as depressive, anxiety and dysbiosis in autism [2], and modulating afferent sensory nerve, gut motility and pain perception [3]. This interaction between gut, microbiota and brain has an important pathological and physiological outcome of such conditions. The disruption of gut-brain axis affects motility and secretion
Received: 11 November 2019; Accepted revised

manuscript: 4 December 2019

Published online: 15 December 2019 leading visceral hypersensitivity, enteroendocrine and immunological imbalance.

Many children have non-specific symptoms and mostly associated to regurgitation, colic, rumination syndrome, functional diarrhoea and cyclical vomiting syndrome. Feeding intolerance, constipation and 'wind' have been blamed in children with severe neurological impairment (SNI) following optimal management of motility disorders. SNI neonates have symptoms such as irritability, hypertonicity, poor weight gain, feeding intolerance which has been attributed to underlying source for neuropathic pain [4]. Even colonic distention produced chronic visceral hypersensitivity, characterised as allodynia, primarily from central neuronal sensitization

\footnotetext{
'Department of Paediatrics, School of Medical Sciences, Universiti Sains Malaysia, Health Campus, 16150 Kubang Kerian, Kelantan, Malaysia

${ }^{2}$ Hospital Universiti Sains Malaysia, 16150 Kubang Kerian, Kelantan, Malaysia

Corresponding author: Dr Fahisham Taib, Department of Paediatrics, School of Medical Sciences, Universiti Sains Malaysia, Health Campus, 16150 Kubang Kerian, Kelantan, Malaysia Tel: +609-7676515; Email: fahisham@gmail.com
} 
without peripheral pathology [5]. Children with SNI have much increased frequency of sensitizing gastrointestinal experiences. Unfortunately, there is no standardized treatment for neuro-enteric dysfunction although various approach has been attempted.

Irritability in non-verbal children may indicate pain. Identifying and scoring of pain can be challenging in these children. Visceral hyperalgesia is often misdiagnosed or not even considered as an aetiology. Visceral hyperalgesia refers to increased perception of pain, arising from the viscera, in response to gastrointestinal sensory stimulus [4]. The diagnosis and treatment options for SNI children with persistent irritability is challenging due to the difficulty to differentiate symptoms of neuropathic pain from other cause of pain. The medical management is often directed toward the neurosensory cause of the symptoms.

Chronic pain in neurologically impaired children In chronic abdominal pain, the complete understanding of the pathophysiology of the functional gastrointestinal disorders remain elusive. Physiological and sociocultural factors may amplify perception of discomfort by caregivers [6]. Symptoms can be severely impacting the daily life activities. Chronic abdominal pain with intestinal inflammation can result in the hyper-responsiveness of neuronal, immune and endocrine signalling pathways within the enteric nervous system (ENS), peripheral nervous system (PNS) and central nervous system (CNS). Gastrointestinal tract inflammation can also cause local hyperexcitability of nociceptor neurons, which persist even after removal of the inflammatory stimulus. Alteration of the neural gut cross-talk and network may be involved in the pathophysiology of functional and inflammatory gastrointestinal disorders. These symptoms are both physiologically multi-determined and modifiable by sociocultural and psychosocial influences [6].

Neuroplastic changes occurred in the brain-gut axis resulting in ongoing chronic abdominal pain. This sensitization may be driven by peripheral mechanisms within the intestinal wall which encompasses an interplay between immunocytes, enterochromaffin cells, resident macrophages, neurons and smooth muscles inflammation and stress. Despite somatic input outnumbering the visceral input, the visceral input still can enter the spinal cord at different spinal segments. Ambiguous signal transduction from the gut to the brain explains why the experienced pain has a poor topological relation and cannot always be pinpointed [7]. ENS comprises enteric nerve cell bodies of sensory, inter- and motor neurons grouped into ganglia interconnected by bundles of nerve processes forming plexuses of which the best characterised are the myenteric plexus (Auerbach's plexus) and the submucosal plexus (Meissner's plexus). When brain encounters stressful life events, the lower gut gets overstimulated resulting in diarrhoea. The extrinsic primary afferents of the gastrointestinal tract provide the anatomical connection with the CNS and hence the basis for both non-painful and painful gut sensations. The gastrointestinal tract receives a dual innervation with complementary roles in gut signalling: a splanchnic and a vagal plus pelvic afferent population. The vagal/pelvic nerves serve homeostatic functions, whereas the splanchnic innervation principally conveys nociception [7].

Children with traumatic brain injury (TBI) has the potential to develop a leaky gut, pointing towards intestinal barrier permeability leak and thus lead to inappropriate signalling of luminal components. The precise mechanism through which TBI leads to these gastrointestinal events is unknown. The systemic physiological effects following TBI, including autonomic dysfunction, systemic inflammation, and organ and gastrointestinal dysfunction, is frequently observed in TBI patients. Intestinal epithelial cells function as a physical barrier between the intestinal lumen and the underlying vasculature and lymphatics. Loss of tight junction barrier integrity has been correlated with increased intestinal permeability, and hypothetically bacterial translocation. Isolated TBI has systemic consequences including pneumonia, cardiovascular disorders, autonomic abnormalities, intestinal dysfunction, multisystem organ failure, gastric ulceration, prolonged ileus, motility problems, and impairment of gut barrier function [8].

\section{Function of enteric nervous system}

ENS is a sophisticated system that able to function independently of central nervous system. The ENS and its neurochemistry resemble central nervous system (CNS), hence pathogenic organism causing CNS disorders may also affect ENS [9]. Gut itself is considered an intricate organ, thus brain-gut axis could be the channel for disease spread. ENS regulates secretion, motility, defence and mucosal maintenance which requires precision degree of control via microcircuits and interneurons. ENS is also phenotypically diverse with the ability to utilise 
various neurotransmitters function with or without CNS input. This signal transits sensation which may not reach consciousness, but influence by the mood instead. Bidirectional signals between the gut and the brain are regulated at neural, immunological and hormonal levels, involving the hypothalamic-pituitaryadrenal axis and the enteric microbiota [6]. However, the crosstalk between gut and brain and mechanism by which the microbiota could alleviate the visceral pain is still in the infancy.

The pathophysiology of disease in CNS affecting ENS covers neurodevelopmental, neurodegenerative and intestinal function. Deficit in ENS may supersede before CNS disease start to manifest. For example, transmissible spongiform encephalopathies such as Creutzfeldt- Jakob disease originated from prion disease entering via gut but eventually manifested after years of incubation. Varicella Zoster Virus (VZV), a highly infectious virus can recur after life-long latency. Reactivation of VZV in ENS may cause gastrointestinal dysfunction without rash. This peripheralization of ENS function has given brain to free brain from connecting to ENS and to allow ENS to work independently [9].

\section{Total pain concept}

Children with SNI experience pain more intensely than neurologically normal children. They are vulnerable to distressing episodes of pain without an identifiable cause. They display altered excitability in the somatosensory cortex. Nociceptive processing at a molecular, cellular and circuit level are altered, leading to wide systemic changes in neuro-excitability that ultimately amplified the pain experience. Nociceptive signals from sensitised spinal afferents are repeatedly received at the dorsal horn of the spinal cord which then lead to a progressive build-up in amplitude of action potentials and cumulative depolarisation known as the 'wind-up phenomenon' [5]. These children continue to experience a steady pain sensation, out of proportion from the original stimulus. Further firing of peripheral nociceptors signal can lead to pain experience that are produced long after the pain stimulus has gone. Non-nociceptive input from other neurones, can also be amplified, resulting in the process of central sensitisation which persists for longer and at greater intensity than is expected.

Damage to the autonomic nervous system is also common in children with SNI. Gastrointestinal dysmotility aggravates further abdominal symptoms such as pain, retching and constipation. Degree of modulation occurs at the point of cognitive and emotional processing, leading to interpretation of non-noxious sensations as noxious. This leads to hypervigilance, thus amplifying further pain experience. Psychosocial and spiritual factors are fundamentally entwined with physical aspects of pain [5]. Other symptoms such as seizures, dystonia and contractures due to spasticity may cause pain, may be triggered by pain from other sources, or become part of the expression of pain behaviours.

Human body has multiple mechanisms to increase or decrease nociceptive input, pain experience and reaction to pain. The mechanisms would involve peripheral and central neuronal activities and long-lasting changes involving neuronal plasticity [10]. This is seen in newborn who experienced transient stimuli but having permanent alteration in experience. Hyperexcitability of neurons from peripheral tissue irritation or in response to influences from the brainstem is the plausible explanation of the ongoing characteristic of chronic pain in these patients.

\section{Available treatment}

The mechanism of action of gabapentin is thought to inhibit or down regulate pain via voltage-dependent calcium ion channels in the central nervous system and relieve the neuropathic pain in response to the gastrointestinal stimulus [4]. Gabapentin has been shown to inhibit central sensitization in a human model of hyperalgesia, reduced rectal sensory thresholds and enhanced rectal compliance in diarrhoea. There are other drugs used for neuropathic pain such as amitryptilline which covers both psychological and physical pain aetiology.

The other approach is to look at the total pain approach. As we understood, most children with SNI have cocktails of causes of pain, and a mixture of nociceptive and neuropathic pain elements. Tackling the psychosocial and spiritual elements are also part of the management of difficult symptom control using a biopsychosocial model approach in the early part of assessment.

The microbiota has emerged as a novel target for treatment of visceral pain. Preclinical evidence showed symptomatology of visceral pain can be reduced but clinical study needs to be confirmed [11]. The improvement of visceral hypersensitivity by using prebiotics and probiotics has been demonstrated but it has lack of power. Faecal 
material transplantation has also gained interest through repopulating new gut microbiota to restore normal gut function [12].

\section{Conclusion}

Often children with SNI has a diffuse, poorly localized and non-specific irritability of chronic pain in the abdomen. The gut-brain neuronal connection has potential roles in development of intestinal dysfunction via the involvement of the brain's emotional limbic system and augmentation of the intestinal stress responses. The final approach should be focused on both physical and psychosocial aspects despite the difficulty in assessment and even intervention in these children.

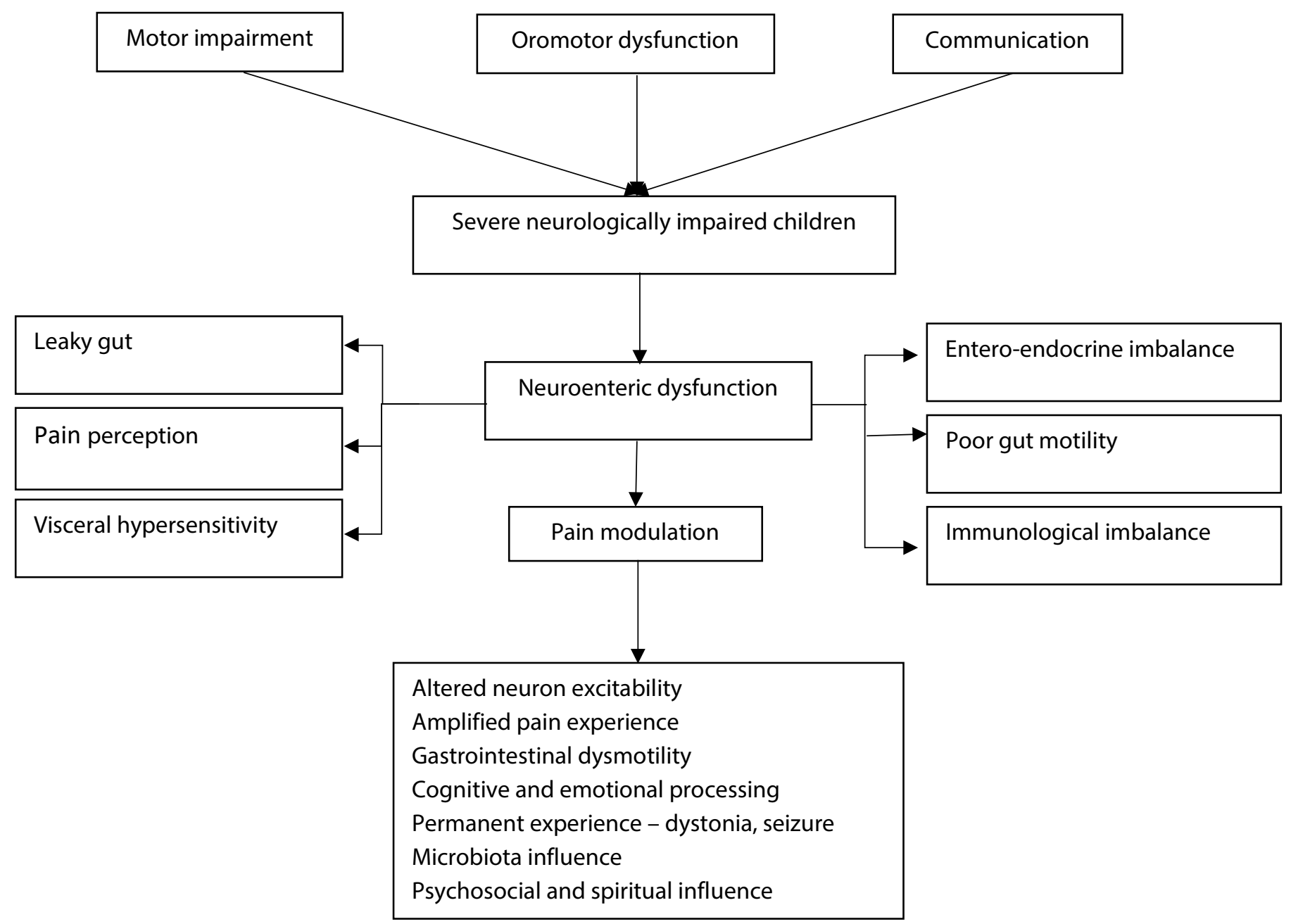

Diagram 1. Factors affecting chronic pain in SNI Children 


\section{References}

[1] Hauer JM, Wical BS, Charnas L. Gabapentin Successfully Manages Chronic Unexplained Irritability in Children with Severe Neurologic Impairment. Pediatrics 2007; 119(2): e519-22

[2] Carabotti M, Scirocco A, Severi C. The gutbrain axis: interaction between enteric and microbiota, centrak and enteric nervous system. Ann Gastroenterol 2015; 28(2): 20309

[3] Ma Q, Xing C, Long W, Wang HY, Liu Q, Wang RF. Impact of microbiota on central nervous system and neurological disease: the gutbrain axis. J Neuroinflammation 2019; 16(1):53

[4] Asaro J, Robinson CA, Levy PT. Visceral Hyperalgesia: When to Consider Gabapentin Use in Neonates-Case Study and Review. Child Neuro Open 2017; 4: 1-6

[5] Warlow T, Hain RDW. Total pain in children with severe neurological impairment. Children 2018; 5: 13

[6] Salvatore S, Barberi S, Borrelli O, Castellazzi A, Di Mauro D, Di Mauro G, Doria M, Francavilla R, Landi M, Martelli A, Miniello VL, Simeone G, Verduci E, Verga C, Zanetti MA, Staiano A and for the SIPPS Working Group on FGIDs. Pharmacological interventions on early functional gastrointestinal disorders. Ital J Pediatr 2016; 42 (1):68

[7] Vermeulen W, De Man JG, Pelckmans PA, De Winter BY. Neuroanatomy of lower gastrointestinal pain disorders. World J Gastroenterol 2014. 28; 20(4): 1005-20

[8] Bansal V, Costantini T, Kroll L, Peterson C, Loomis W, Eliceiri B, Baird A, Wolf $P$, Coimbra R. Traumatic Brain Injury and Intestinal Dysfunction: Uncovering the Neuro-Enteric Axis. J Neurotrauma 2009; 26:1353-59

[9] Rao M, Gershon MD. The bowel and beyond: the enteric nervous system in neurological disorders. Nat Rev Gastroenterol Hepatol 2016; 13: 517-28

[10] Mayer EA, Gebhart GF. Basic and clinical aspects of visceral hyperalgesia. Gastroenterology 1994: 107; 271-93

[11] Pusceddu MM, Gareau MG. Visceral pain: gut microbiota, a new hope? J Biomed Sci 2018; 25: 73

[12] Kim KK, Gluck M. Fecal Microbiota Transplantation: An Update of Clinical Practice. Clin Endosc 2019; 52: 137-43 